\title{
Les traits de personnalité et le succès académique en marketing et en comptabilité
}

\author{
Stéphane Aubin, D. Sc. (gestion) et Sanaâ El Boukri \\ Université du Québec à Chicoutimi
}

\section{INTRODUCTION}

\section{Depuis toujours, la psychologie du travail cherche à identifier chez l'humain ce qui peut contribuer à améliorer l'efficacité productive (quantité, qualité et délai de production d'un bien ou d'un service) et l'efficacité psycho-sociale (climat, engagement et éthique).}

Depuis toujours, la psychologie du travail cherche à identifier chez l'humain ce qui peut contribuer à améliorer l'efficacité productive (quantité, qualité et délai de production d'un bien ou d'un service) et l'efficacité psycho-sociale (climat, engagement et éthique). À ces fins, différentes méthodes de sélection ont été développées pour optimiser la dotation, telles que l'utilisation de tests, d'entrevues et de mises en situation visant à assigner le meilleur candidat à un poste. Ces épreuves permettent d'évaluer les capacités et la motivation des candidats. Considérant que la personnalité est présumée stable dans le temps et dans diverses situations, on croit qu'elle peut constituer un déterminant utile pour prévoir l'efficacité ${ }^{1}$, la satisfaction et le succès des personnes au travail ${ }^{2}$. Par ailleurs, des recherches établissent un continuum entre les dispositions personnelles, le succès académique et la performance au travail ${ }^{3}$.

Certaines caractéristiques personnelles, la personnalité, les intérêts et les valeurs notamment, ont de plus grandes possibilités de s'exprimer dans certains types d'environnement: la congruence entre les types de personnalité et d'environnement favorise la satisfaction au travail, la stabilité du cheminement de carrière et la performance au travail ${ }^{2}$.

Dès l'adolescence, les personnes ont à faire des choix vocationnels; elles s'intéressent aux sciences exactes, à la relation d'aide, à l'informatique, à la politique ou à la création artistique, par exemple. Elles décident alors d'entreprendre des études dans le domaine qui leur parait le plus attrayant. $\mathrm{Ce}$ processus relève de la conviction que le programme de formation choisi favorisera l'accès à des professions et des milieux de travail où elles pourront exercer des activités qui leur plaisent, avoir accès à des «récompenses » valorisées et résoudre des problèmes correspondant à leurs compétences. Après quelque temps, certaines voient leurs attentes comblées alors que d'autres doivent réévaluer leur choix; ce n'est pas ce à quoi elles s'attendaient, elles ont de la difficulté à bien réussir les épreuves ou n'aiment pas «le genre de personnes» qu'on retrouve dans ce programme.

Un type d'emploi donné regroupe des gens qui se ressemblent en partie tant au point de vue de leur histoire personnelle que de leur personnalité.

Selon Holland ${ }^{2}$, un type d'emploi donné regroupe des gens qui se ressemblent en partie tant au point de vue de leur histoire personnelle que de leur personnalité. Ainsi, la manière dont ces personnes abordent les problèmes et dont elles interagissent présente des similitudes. Les milieux académiques et de travail ont tendance à devenir de plus en plus homogènes par l'attraction et la sélection des candidats qui ont un profil correspondant à certaines normes et le rejet des membres qui s'en éloignent ${ }^{4}$.

Peu d'études se sont intéressées à clarifier si les étudiants qui ont un profil personnel correspondant aux stéréotypes professionnels réussissent mieux que les autres et s'ils sont plus satisfaits de leurs études. Notre enquête vise à répondre à ces questions pour les cas des études universitaires en marketing et en comptabilité. 


\section{PERSONNALITÉ ET RENDEMENT AU TRAVAIL}

À partir de 1995, comme l'illustre la figure 1, le nombre de publications reliant la personnalité et le rendement au travail a sensiblement augmenté. Ces données sont le résultat d'une recher- che dans APA PsycNet qui identifie le nombre d'articles publiés dans des revues spécialisées dans lesquels les mots personality et jobperformance sont mentionnés dans les résumés.

Figure 1 - Évolution du nombre de résumés d'articles reliant personnalité et rendement au travail (1960-2009)

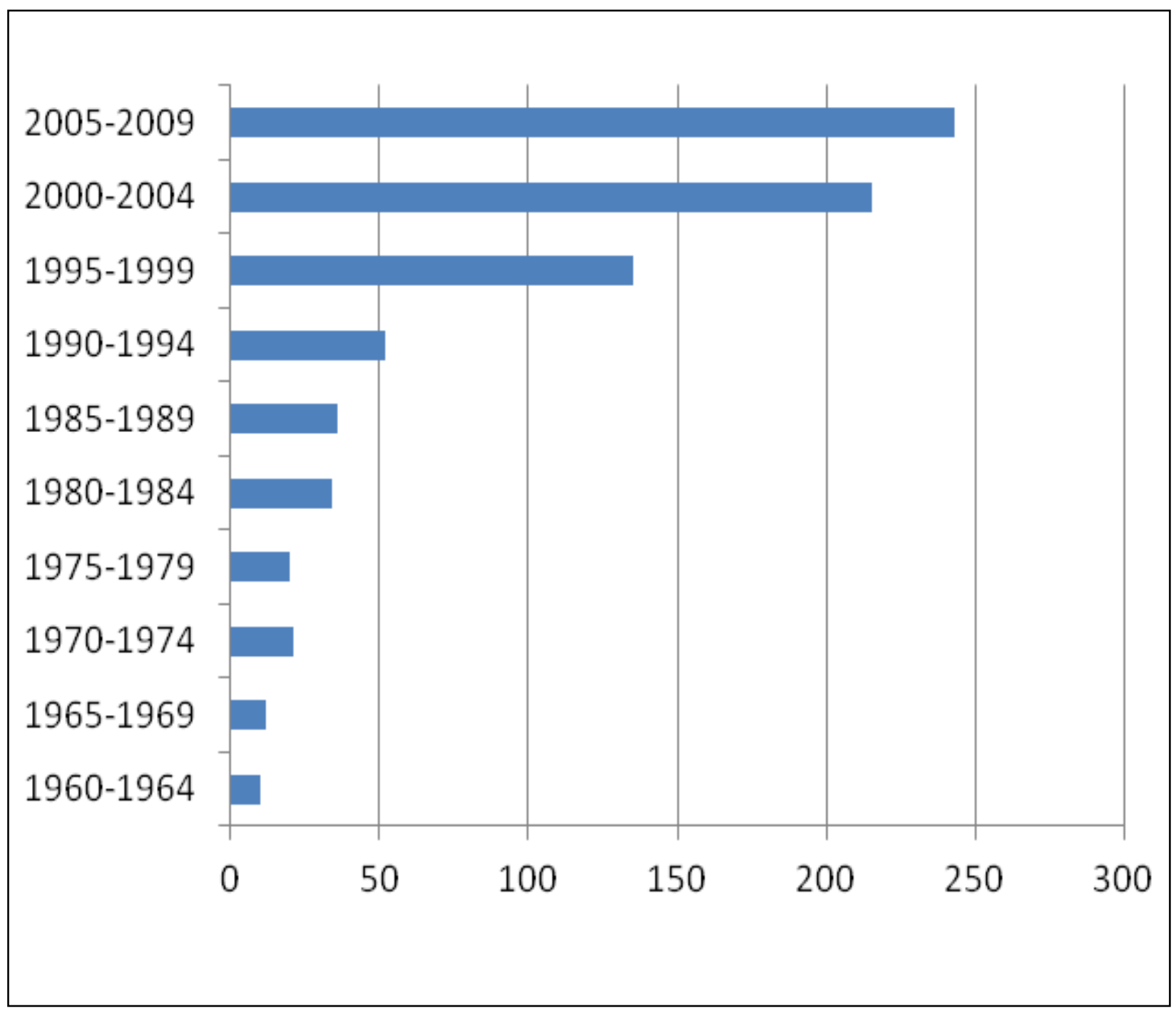

Ce sujet de recherche a connu un regain d'intérêt suite aux publications de Costa et $\mathrm{McCrae}^{5}$ au début des années 90 . Ce point tournant a été la formalisation d'un modèle qui définit la personnalité par cinq grandes dimensions: l'extraversion, le névrosisme, l'ouverture à l'expérience, l'amabilité et la fiabilité (les Big Five, selon l'expression consacrée). Chacune de celles-ci se situe sur un axe; une personne en particulier présente un certain degré d'extraversion par exemple. Le tableau 1 présente les principales caractéristiques associées à chacune des extrémités du continuum de ces dimensions. 
Tableau 1 - Caractéristiques des individus pour chacune des cinq grandes dimensions

\begin{tabular}{|c|c|}
\hline $\begin{array}{l}\text { Extraversion } \\
\text { L'individu extraverti est optimiste, grégaire, } \\
\text { amical, sociable, ambitieux et loquace }\end{array}$ & $\begin{array}{l}\text { Introversion } \\
\text { Celui qui est peu extraverti est plutôt réservé, } \\
\text { sobre, discret, solitaire et réfléchi }\end{array}$ \\
\hline $\begin{array}{l}\text { Amabilité } \\
\text { La personne aimable est serviable, indulgente, } \\
\text { crédule, franche, douce, gentille, } \\
\text { accommodante, sympathique, cordiale, } \\
\text { chaleureuse et sincère }\end{array}$ & $\begin{array}{l}\text { Hostilité } \\
\text { Celle qui ne l'est pas est plus cynique, } \\
\text { impolie, méfiante, vindicative, irritable, } \\
\text { manipulatrice, antagoniste, entêtée, } \\
\text { confrontante et impitoyable }\end{array}$ \\
\hline $\begin{array}{l}\text { Fiabilité } \\
\text { Les gens fiables sont soignés, travailleurs, } \\
\text { ponctuels, scrupuleux, méthodiques, } \\
\text { méticuleux, économes et persévérants }\end{array}$ & $\begin{array}{l}\text { Insouciance } \\
\text { À l'inverse, les insouciants sont désorganisés, } \\
\text { extravagants, négligents, paresseux, débraillés, } \\
\text { en retard et étourdis }\end{array}$ \\
\hline $\begin{array}{l}\text { Stabilité émotionnelle } \\
\text { La personne émotionnellement stable est } \\
\text { calme, non-envieuse, patiente, détendue, } \\
\text { robuste, tranquille et satisfaite }\end{array}$ & $\begin{array}{l}\text { Névrosisme } \\
\text { L'instabilité émotionnelle se manifeste par } \\
\text { l'inquiétude, la nervosité, l'émotivité, } \\
\text { l'inadaptation, l'hypocondrie, la colère, } \\
\text { l'anxiété, la jalousie et l'agitation }\end{array}$ \\
\hline $\begin{array}{l}\text { Ouverture à l'expérience } \\
\text { L'individu ouvert est artiste, créatif, } \\
\text { imaginatif, compliqué, intense, idéaliste, } \\
\text { informé, articulé, intelligent, ingénieux, } \\
\text { curieux, éclectique, original et non- } \\
\text { conformiste }\end{array}$ & $\begin{array}{l}\text { Fermeture à l'expérience } \\
\text { Celui qui n'est pas ouvert est plutôt ordinaire, } \\
\text { terre à terre, direct, prévisible, superficiel, } \\
\text { laconique, non-perspicace, conformiste, } \\
\text { réaliste et exclusif }\end{array}$ \\
\hline
\end{tabular}

Les recherches qui visent à établir des liens entre les Big Five d'une part, et les attitudes et le rendement au travail d'autre part concluent que :

- Considérées ensemble, ces cinq grandes dimensions de la personnalité sont associées au rendement au travail, aux comportements contreproductifs, à la citoyenneté organisationnelle, à la sociabilité, au travail d'équipe, à la motivation, au leadership et à la satisfaction au travail ${ }^{6}$;

- La Fiabilité est la dimension la plus souvent citée comme déterminant du rendement au travail ${ }^{1,6,7}$ pour tous les types d'emplois;

- Pour les emplois spécialisés et semi-spécialisés, la Stabilité émotionnelle est aussi liée au rendement ${ }^{8}$;

- Le rendement du personnel de direction est corrélé à l'Extraversion ${ }^{7,9}$.

Un autre modèle, partiellement dérivé du Big Five, propose quatre caractéristiques pouvant expliquer le rendement et la satisfaction au travail. Le noyau de l'évaluation de soi (Core Self-Evaluation ou Positive self-concept) réfère aux évaluations fondamentales que les personnes font à propos de leurs qualités, compétences et potentiel ${ }^{10}$. Il est constitué des traits suivants: l'estime de soi, le sentiment d'efficacité personnelle généralisée, la stabilité émotionnelle et le locus de contrôle. Les recherches ${ }^{11}$ sur ce modèle indiquent que :

- Ces quatre traits sont autant associés à la satisfaction et au rendement au travail que le sont les Big Five;

- Conjointement, les traits sont corrélés au rendement au même niveau que l'est la Fiabilité;

- Quand on les considère un par un, les coefficients de Pearson varient de .24 à .45 avec la satisfaction au travail et de .19 à .26 avec le rendement

Le tableau 2 résume les conclusions d'études sur les liens entre les traits de personnalité et le rendement au travail ${ }^{1}$. 
Tableau 2 - Liens entre traits de personnalité et performance

\begin{tabular}{|c|c|}
\hline & Rendement au travail \\
\hline Extraversion & $\begin{array}{l}\text { Associée à la performance dans les domaines de la vente et } \\
\text { de la gestion et pour le travail d'équipe. Le lien est cependant } \\
\text { modéré par le degré de stabilité émotionnelle }{ }^{11} \text {. }\end{array}$ \\
\hline Fiabilité & $\begin{array}{l}\text { C'est la dimension la plus souvent citée comme étant } \\
\text { associée à la performance au travail }{ }^{12,13} \text {. Cette relation est } \\
\text { cependant modulée par le degré d'amabilité }: \text { les personnes } \\
\text { qui ont des scores élevés pour la Fiabilité et faibles pour } \\
\text { l'Amabilité peuvent paraitre rigides, inflexibles ou trop axées } \\
\text { sur la tâche. Par contre, les équipiers apprécient leur sens de } \\
\text { l'organisation et les règles de fonctionnement qu'ils } \\
\text { établissent }^{1} \text {; }\end{array}$ \\
\hline Ouverture à l'expérience & $\begin{array}{l}\text { Favorable en contexte d'entrainement à la tâche ou de } \\
\text { formation, l'ouverture est peu liée à la performance. }\end{array}$ \\
\hline Stabilité émotionnelle & $\begin{array}{l}\text { Prise isolément, la Stabilité émotionnelle a une relation faible } \\
\text { ou modérée avec la performance au travail. Elle semble } \\
\text { davantage agir comme catalyseur en appuyant d'autres } \\
\text { facteurs, l'Esprit consciencieux et l'Extraversion notamment, } \\
\text { dans leur effet bénéfique sur le fonctionnement des équipes } \\
\text { et sur les relations interpersonnelles en général. }\end{array}$ \\
\hline Amabilité & Il y a peu de liens directs mentionnés dans la littérature. \\
\hline Locus of control & $\begin{array}{l}\text { Corrélation modérée avec la performance et la satisfaction } \\
\text { au travail. }\end{array}$ \\
\hline Sentiment d'efficacité & $\begin{array}{l}\text { Corrélation modérée avec la performance et élevée }(0,45) \\
\text { avec la satisfaction au travail }{ }^{11} \text {. }\end{array}$ \\
\hline Estime de soi & $\begin{array}{l}\text { Les résultats de recherches concernant l'Estime de soi } \\
\text { divergent; certaines concluent qu'elle est liée à la } \\
\text { performance et à la satisfaction au travail }{ }^{11} \text { alors que d'autres } \\
\text { affirment le contraire ou que la causalité est inverse; la } \\
\text { performance augmente l'estime de soi }{ }^{14} \text {. }\end{array}$ \\
\hline
\end{tabular}

\section{PERSONNALITÉ ET PROFESSION}

Selon le modèle Attraction-Sélection-Attrition (ASA) de Schneider ${ }^{4}$, les milieux de travail ont tendance à présenter une homogénéité de plus en plus grande avec le temps. Ce processus d'uniformisation procède en trois phases résumées dans l'acronyme du modèle.

Premièrement, en ce qui concerne l'Attraction, un milieu de travail donné présente certaines caractéristiques réelles ou apparentes qui favorisent le développement de stéréotypes professionnels relativement stables. On considère par exemple les comptables plus organisés et prévisibles que les artistes. Les comptables travaillent souvent seuls et ont comme environnement de travail un bureau assez traditionnel (chaise, table de travail, classeur). Ils travaillent avec des données qu'ils ont à classer et à interpréter selon certaines normes. Les artistes, eux, utilisent davantage leur imagination, travaillent dans des environnements diversifiés, ont des revenus d'emploi variables et sont plus rarement que les comptables au service de grandes entreprises.

Les personnes qui ont des valeurs, des intérêts et des habiletés qui correspondent à ceux d'un milieu de travail donné seraient plus tentées d'en faire partie. Elles espèrent ainsi pouvoir s'exprimer, se réaliser et y trouver des avantages et récompenses correspondant à leurs préférences. 
Deuxièmement, pour la sélection, les organisations et les personnes qui les composent valorisent des caractéristiques particulières : être méticuleux, avoir des valeurs économiques fortes, être relativement conformiste peut paraître «normal» pour des comptables mais moins important pour des artistes. Les groupes professionnels se dotent de processus pour recruter les meilleurs talents, souvent on les reconnaît à travers leurs valeurs et intérêts. Il en va de même pour la culture des groupes; dans un cas on valorise la prospérité (les comptables) et dans l'autre, l'originalité (les artistes), par exemple. Cette phase de tamisage élimine les candidats qui ne partagent pas les valeurs, intérêts et compétences traditionnels ou attendus. Cela contribue à l'homogénéisation des groupes professionnels.

Finalement, pour expliquer le troisième $\mathrm{A}$ de l'acronyme, il arrive que certains membres de groupes professionnels qui ont été attirés et sélectionnés constatent qu'ils ne se plaisent pas dans le contexte qu'ils avaient cru plus propice à l'expression de leur individualité, qu'ils ne reçoivent pas les récompenses attendues ou ne sont pas appréciés à leur valeur. Parfois, c'est l'organisation qui s'aperçoit qu'ils ne font pas l'affaire (valeurs, compétences ou motivations non-alignées avec celles du groupe, par exemple). C'est la phase d'Attrition; les groupes professionnels se départent des individus « déviants », ce qui boucle la boucle de l'uniformisation des regroupements professionnels.

Pour expliquer ces regroupements, ces personnalités collectives, Holland ${ }^{2}$ propose une typologie vocationnelle. Selon lui, le choix d'une profession représente l'expression de la personnalité. L'individu exprime par ce choix ses motivations, ses connaissances, un mode de vie souhaité, l'emploi d'outils, la recherche d'un statut et d'un rôle social et des aptitudes. Ainsi, les stéréotypes que les gens entretiennent au sujet des professions sont en grande partie valides. Un certain consensus existerait dans la société à ce sujet, les gens partageant les mêmes idées et sentiments vis-à-vis d'occupations particulières. Ces perceptions sont d'ailleurs relativement stables. Un type d'emploi donné regroupe également des gens qui se ressemblent en partie tant au point de vue de leur histoire personnelle que de leur personnalité. Ainsi, la manière dont ces personnes abordent les problèmes et dont elles interagissent présente des similitudes. Enfin, la satisfaction, la stabilité et le succès sur le plan professionnel dépendent de la congruence entre la personnalité de l'individu et l'environnement dans lequel il travaille. Dans plusieurs situations, l'environnement est constitué de personnes.

Holland décrit six types de personnalité : le Réaliste, l'Investigateur, l'Artistique, le Social, l'Entreprenant et le Conventionnel. Les regroupements de personnes partageant les caractéristiques de ces types favorisent l'émergence d'environnements correspondants.

En ce qui concerne notre étude, nous nous attarderons sur deux types de personnalité : l'entreprenant et le conventionnel car, comme on le verra, ils correspondent aux personnes occupant des emplois dans le domaine du marketing et de la comptabilité.

\section{Les gens du type Entreprenant aiment bien la compagnie et le contact humain. Cependant, la finalité de ces relations est l'atteinte de buts organisationnels et économiques par la persuasion. Les domaines de la vente, l'exploitation commerciale, la gestion, le droit et la politique peuvent s'avérer propices à fournir le genre de résultats recherchés.}

Les gens du type Entreprenant aiment bien la compagnie et le contact humain. Cependant, la finalité de ces relations est l'atteinte de buts organisationnels et économiques par la persuasion. Les domaines de la vente, l'exploitation commerciale, la gestion, le droit et la politique peuvent s'avérer propices à fournir le genre de résultats recherchés. Ces personnes ont de la facilité à s'exprimer, ce qui leur permet d'avoir accès à des emplois nécessitant du leadership ou à assumer la direction de personnel. Elles sont reconnues comme étant énergiques, aventureuses, sociables, ambitieuses, impulsives, sûres 
d'elles, optimistes, autoritaires, populaires, extraverties, exhibitionnistes et loquaces. Le travail de précision, l'effort intellectuel prolongé, les activités symboliques, systématiques et d'observation les rebutent. La politique et la réussite au plan financier sont parmi les valeurs auxquelles elles sont attachées.

\section{La personne de type Conventionnel préfère les activités nécessitant la manipulation systématique, explicite et ordonnée de données selon un plan prescrit. La comptabilité, les emplois dans les banques, l'expertise fiscale, la consignation de données testamentaires, la mesure du risque actuariel sont des emplois recherchés par la personne Conventionnelle.}

Les personnes appartenant au type Conventionnel partagent avec l'Entreprenant un intérêt envers les activités à caractère économique. Cependant, ce n'est pas de la même façon qu'elles cherchent à arriver à leurs fins. Ainsi la personne de type Conventionnel préfère les activités nécessitant la manipulation systématique, explicite et ordonnée de données selon un plan prescrit.

La comptabilité, les emplois dans les banques, l'expertise fiscale, la consignation de données testamentaires, la mesure du risque actuariel sont des emplois recherchés par la personne Conventionnelle. Elle est souvent décrite comme étant compétente, inhibée, persévérante, conformiste, calme, peu imaginative, consciencieuse, méthodique, disciplinée, pratique, conservatrice, obéissante, soignée, efficace, prude, inflexible et économe.

Pour travailler dans les conditions souhaitées, les personnes de type Conventionnel seraient attirées par les grandes organisations sans pour autant rechercher des postes de commande. Ces personnes admettent être inconfortables dans des situations ambiguës dans lesquelles elles souffrent de ne pas savoir ce qui est attendu d'elles. Douées pour les mathématiques et le travail de bureau, elles ont peu de talent pour les arts. Elles partagent avec les Entreprenants des valeurs économiques.
On peut penser que les emplois dans le domaine du marketing sont occupés par des personnes extraverties (optimistes, grégaires, amicales, sociables, ambitieuses et loquaces) alors que ceux en comptabilité le sont par des personnes fiables (soignées, travaillantes, ponctuelles, scrupuleuses, méthodiques, méticuleuses, économes et persévérantes).

Une étude ${ }^{16}$ sur la correspondance entre les Big Five et les types de personnalité souligne des liens significatifs entre le type Entreprenant et l'extraversion (.50), la Fiabilité (.28) et la Stabilité émotionnelle (.24) alors que le type Conventionnel présente un lien inverse (-.20) avec l'Ouverture à l'expérience. D'une autre façon, les analyses indiquent que les personnes extraverties sont entreprenantes (.56) alors que les gens fiables sont du type conventionnel (.35).

En résumé, on peut penser que les emplois dans le domaine du marketing sont occupés par des personnes extraverties (optimistes, grégaires, amicales, sociables, ambitieuses et loquaces) alors que ceux en comptabilité le sont par des personnes fiables (soignées, travaillantes, ponctuelles, scrupuleuses, méthodiques, méticuleuses, économes et persévérantes).

Le cheminement académique devrait se situer en continuité avec le milieu de travail visé. Les cours et les programmes devraient valoriser les caractéristiques particulières des milieux où s'exercent les types de professions reliées : la créativité de l'artiste, l'éloquence du politicien et de l'avocat plaideur, la rigueur du scientifique, l'humanisme du travailleur social, par exemple.

Considérant cela, le cheminement académique devrait se situer en continuité avec le milieu de travail visé. Les cours et les programmes devraient valoriser les caractéristiques particulières des milieux où s'exercent les types de professions reliées: la créativité de l'artiste, l'éloquence du politicien et de l'avocat plaideur, la rigueur du scientifique, l'humanisme du travailleur social, par exemple. Mais est-ce vraiment le cas? Est-ce que le meilleur étudiant dans un domaine réussit mieux les épreuves de sélection que les autres? A-t-il plus de succès professionnel? 


\section{PERSONNALITÉ ET RENDEMENT ACADÉMIQUE}

Une méta-analyse ${ }^{17}$ sur les liens entre les dimensions du Big Five et le succès académique (notes, persévérance et satisfaction) indique que la Stabilité émotionnelle est liée à la satisfaction $(0,369)$ alors que la Fiabilité est corrélée $(0,269)$ aux résultats académique. Les trois autres dimensions, Extraversion, Ouverture et Amabilité, pour leur part, ne présentent pas de liens significatifs avec le succès académique. Une autre étude ${ }^{18}$ conclut que la Stabilité émotionnelle et la Fiabilité sont systématiquement associées aux résultats académiques (examens et projets de fin d'études). L'Ouverture est parfois mentionnée ${ }^{19}$ comme étant liée au succès académique. En ce qui concerne l'Extraversion, on ne signale qu'une faible relation inverse (-.059) avec les résultats académiques; les corrélations varient cependant entre -.32 et .78 , ce qui rend toute interprétation périlleuse.

\section{La relation entre l'Extraversion et le succès académique est plus ambiguë. Les recherches sur la question ne l'identifient pas systématiquement comme étant associée au succès académique. Ce facteur caractérise davantage les étudiants populaires et fêtards que studieux et brillants.}

En résumé, le facteur Fiabilité devrait favoriser le rendement académique dans tous les domaines, peut-être même davantage en comptabilité où les environnements de travail des professionnels de ce domaine valorisent les caractéristiques y étant associées (voir tableau 1). La relation entre l'Extra-

\section{MÉTHODOLOGIE}

Un questionnaire de 37 items tirés du Core Self Evaluation Scale ${ }^{20}$ et de l'International Personality Item Pool ${ }^{21}$ a été distribué à 289 étudiants du baccalauréat en sciences administratives à l'Université du Québec à Chicoutimi inscrits à un cours en marketing ou en comptabilité; de ceux-là, 217 l'ont rempli et remis (53\% de sexe féminin, $52 \%$ en comptabilité). Les sujets avaient à indiquer leur degré d'accord avec des propositions relatives aux neuf variables, soit l'extraversion, la fiabilité, la stabilité émotionnelle, l'estime de soi, le sentiment d'efficacité, le locus de contrôle, l'intelligence version et le succès académique est plus ambiguë. Les recherches sur la question ne l'identifient pas systématiquement comme étant associée au succès académique. Ce facteur caractérise davantage les étudiants populaires et fêtards que studieux et brillants. Cependant, il existe un certain consensus quant au lien entre l'Extraversion et le rendement au travail dans les domaines de la vente et de la gestion $^{7,8,9}$.

L'objectif de notre recherche est de mieux comprendre comment le processus ASA se manifeste à l'université et d'identifier ce qui contribue au succès académique dans les domaines du marketing et de la comptabilité. Nous désirons vérifier si les stéréotypes se vérifient et jusqu'à quel point ils s'observent.

Au départ, nous avons cinq hypothèses à vérifier. Premièrement, certains traits de personnalité (parmi la Stabilité émotionnelle, l'Estime de soi, le Locus de contrôle, le Sentiment d'efficacité et l'Intelligence émotionnelle) sont associés au succès académique. Deuxièmement, la Fiabilité est positivement corrélée aux résultats académiques en comptabilité et en marketing. Troisièmement, l'Extraversion est positivement corrélée aux résultats académiques en marketing et négativement corrélée en comptabilité. Quatrièmement, la satisfaction générale et la satisfaction aux études sont associées à la Stabilité émotionnelle. Cinquièmement, il existe un lien entre la satisfaction générale et la satisfaction aux études et la performance académique.

émotionnelle, la satisfaction de vie et la satisfaction pour les études. La variable dépendante est le résultat académique (la note).

Nous avons calculé les alphas de Cronbach pour chacune des échelles (ils s'établissent entre .64 et .82 , ce qui est très satisfaisant), procédé à une analyse factorielle par composantes principales (extraction de trois facteurs expliquant $37 \%$ de la variance observée : sociabilité, ordre et rigueur et confiance en soi) et fait l'analyse de régression pour l'ensemble des variables. 


\section{RÉSULTATS}

Parmi les variables identifiées dans la première hypothèse, seul le sentiment d'efficacité présente un lien significatif avec le succès académique $(.23, \mathrm{p}<.01)$. Les autres affichent les corrélations modestes qui varient entre -.04 et .10.

Les résultats appuient la seconde hypothèse. En effet, la Fiabilité est significativement associée au résultat académique (.18, p<.05). Il en va de même pour le facteur Ordre et rigueur (qui regroupe cinq des dix items reliés à la mesure de la Fiabilité). Ces résultats s'appliquent tant au groupe en comptabilité qu'à celui en marketing.

La troisième hypothèse propose des liens contraires entre l'Extraversion et les résultats en comptabilité d'une part et une relation positive en marketing, d'autre part. De façon générale, pour tous les étudiants, il y a peu de liens (-.07, non-significatif) entre le degré d'Extraversion et les résultats académiques. Cette même tendance s'observe chez les étudiants en marketing: les résultats obtenus au plan académique ne varient pas conjointement avec le degré d'extraversion des sujets. Il en va de même avec les étudiants en comptabilité.

Pour la quatrième hypothèse, nous n'observons pas de liens importants entre la stabilité émotionnelle et les autres variables étudiées; la satisfaction générale (.08, non-significatif) et la satisfaction des études $(.16, \quad \mathrm{p}<.05)$ en particulier.

Enfin, en ce qui concerne la cinquième hypothèse, les résultats divergent. La satisfaction générale est faiblement liée aux résultats (.05, non-significatif) alors que la satisfaction pour les études (comme étudiant, je suis satisfait) est la variable la plus associée avec le succès académique $(.29, \mathrm{p}<.001)$. Le tableau 3 présente les moyennes, écarts-types et corrélations entre les variables étudiées ${ }^{22}$. Parmi ces données, il ressort des conclusions significatives.

Premièrement, l'Extraversion et la satisfaction générale, l'estime de soi et l'intelligence émotionnelle sont très corrélées. L'optimisme et la sociabilité de l'extraverti peuvent expliquer ces liens.
Deuxièmement, une corrélation forte existe entre la Fiabilité et le sentiment d'efficacité, l'estime de soi, la satisfaction des études et les résultats académiques. L'esprit méthodique et la persévérance de la personne fiable peuvent contribuer au développement de ces attitudes de considération favorable de son efficacité, d'ellemême et de ses études et, parallèlement, favoriser l'atteinte de résultats supérieurs.

\section{C'est la conviction d'avoir une influence sur sa vie et de pouvoir en tracer le destin qui peut être associé à la perception d'être responsable des résultats. Un biais auto- avantageux favorise la considération positive que l'individu interne porte à sa personne, à ses relations, à sa vie et à ses études.}

Troisièmement, le Locus de contrôle est significativement corrélé à la satisfaction générale, au sentiment d'efficacité, à l'estime de soi, à la satisfaction des études et à l'intelligence émotionnelle. Ici, c'est la conviction d'avoir une influence sur sa vie et de pouvoir en tracer le destin qui peut être associé à la perception d'être responsable des résultats. Un biais autoavantageux favorise la considération positive que l'individu porte à sa personne, à ses relations, à sa vie et à ses études.

Quatrièmement, le Sentiment d'efficacité est très relié à l'estime de soi à la satisfaction des études comme des recherches antérieures ${ }^{10} 1$ 'ont rapporté. Il est aussi corrélé à l'intelligence émotionnelle. Curieusement, il affiche peu de lien avec la Stabilité émotionnelle alors que Judge ${ }^{23}$ rapporte un lien étroit entre ces deux variables.

Enfin, l'estime de soi affiche des taux de corrélation relativement élevés avec la satisfaction des études et l'intelligence émotionnelle.

Le Sentiment d'efficacité est très relié à l'estime de soi à la satisfaction des études comme des recherches antérieures l'ont rapporté. Il est aussi corrélé à l'intelligence émotionnelle. 
Tableau 3 - Moyennes, écarts-types et corrélations entre les variables ${ }^{22}$

\begin{tabular}{|c|c|c|c|c|c|c|c|c|c|c|c|c|}
\hline & $\mathrm{M}$ & ÉT & 1 & 2 & 3 & 4 & 5 & 6 & 7 & 8 & 9 & 10 \\
\hline $\begin{array}{l}1- \\
\text { Extraversion }\end{array}$ & 3.63 & .63 & .81 & & & & & & & & & \\
\hline 2- Fiabilité & 3.74 & .65 & $-.22^{\mathrm{a}}$ & .82 & & & & & & & & \\
\hline $\begin{array}{l}\text { 3-Locus de } \\
\text { contrôle }\end{array}$ & 4 & .71 & $.18^{\mathrm{a}}$ & $.21^{\mathrm{b}}$ & .23 & & & & & & & \\
\hline $\begin{array}{l}4- \\
\text { Satisfaction } \\
\text { générale }\end{array}$ & 4.05 & 1.12 & $.33^{\mathrm{b}}$ & $.18^{\mathrm{b}}$ & $.33^{\mathrm{b}}$ & .86 & & & & & & \\
\hline $\begin{array}{l}\text { 5-Sentiment } \\
\text { d'efficacité }\end{array}$ & 4.03 & .63 & .08 & $.50^{\mathrm{c}}$ & $.35^{\mathrm{c}}$ & $.34^{\mathrm{b}}$ & .64 & & & & & \\
\hline $\begin{array}{l}\text { 6-Estime de } \\
\text { soi }\end{array}$ & 4.23 & .54 & $.33^{\mathrm{c}}$ & $.26^{\mathrm{c}}$ & $.49^{\mathrm{c}}$ & $.30^{\mathrm{c}}$ & $.54^{\mathrm{c}}$ & .62 & & & & \\
\hline $\begin{array}{l}\text { 7-Stabilité } \\
\text { émotionnelle }\end{array}$ & 2.86 & .70 & -.09 & -.09 & -.03 & -.08 & -.13 & $-.14^{\mathrm{a}}$ & .200 & & & \\
\hline $\begin{array}{l}8- \\
\text { Satisfaction } \\
\text { des études }\end{array}$ & 3.77 & .88 & .09 & $.29^{\mathrm{c}}$ & $.32^{\mathrm{c}}$ & $.16^{\mathrm{a}}$ & $.33^{\mathrm{c}}$ & $.40^{\mathrm{c}}$ & .07 & 1 & & \\
\hline $\begin{array}{l}9 \\
\text { Intelligence } \\
\text { émotionnelle }\end{array}$ & 4.16 & .75 & $.33^{\mathrm{c}}$ & -.08 & $.28^{\mathrm{c}}$ & $.30^{\mathrm{c}}$ & $.29^{\mathrm{c}}$ & $.35^{\mathrm{c}}$ & .06 & .13 & 1 & \\
\hline $\begin{array}{l}10 \text { Résultats } \\
\text { académiques } \\
\text { (note) }\end{array}$ & $\mathrm{X}$ & $\mathrm{X}$ & -.07 & $.18^{\mathrm{a}}$ & .09 & .05 & $.23^{\mathrm{b}}$ & .10 & -.04 & $.29^{\mathrm{c}}$ & -.09 & 1 \\
\hline
\end{tabular}

${ }^{\mathrm{a}}: \mathrm{p}<.05,{ }^{\mathrm{b}}: \mathrm{p}<.01,{ }^{\mathrm{c}}: \mathrm{p}<.001$. Les nombre de la diagonale représentent les coefficients alpha de Cronbach (sauf Estime de soi et sentiment d'efficacité où ce sont les coefficients de corrélation qui sont mentionnés)

\section{ANALYSE DES RÉSULTATS}

Les résultats relatifs à la première hypothèse indiquent qu'il existe effectivement un lien entre les variables du noyau de l'évaluation de soi et les résultats académiques; il s'agit plus précisément du sentiment d'efficacité mesuré à l'aide des trois énoncés suivants : 1) Je termine ce que je commence, 2) Quand je fais des plans, je suis à peu près sûr de les réaliser et 3 ) Je réussis ce que j'entreprends. Ces mesures sont de nature nettement plus conatives que celles des autres variables du noyau telles que : je connais mes forces, je m'aime comme je suis, je sens que je suis une personne de valeur, je sens que je n'ai pas grand-chose dont je peux être fier, et autres. Le sentiment d'efficacité est relié directement à la réalisation de tâches et de mandats, ce qui le rapproche de la variable Fiabilité.

Les caractéristiques des personnes « fiables » (soignées, travailleuses, ponctuelles, scrupuleuses, méthodiques, méticuleuses, économes et persévérantes) sont associées à un bon rendement tant au plan professionnel qu'académique.
Comme prévu, la Fiabilité présente une relation significative avec la performance (aux études dans notre cas). Les caractéristiques des personnes « fiables » (soignées, travailleuses, ponctuelles, scrupuleuses, méthodiques, méticuleuses, économes et persévérantes) sont associées à un bon rendement tant au plan professionnel qu'académique, ce qui n'est pas étonnant. Pour obtenir de bons résultats, les étudiants doivent assister aux cours, être attentifs, faire les lectures, avoir de bonnes méthodes d'étude et être bien organisés, ce qui semble aller de soi.

L'Extraversion ne constitue pas un trait favorable au succès aux études. Si dans les emplois dans lesquels il y a beaucoup de relations interpersonnelles comme la vente et la gestion, ce facteur peut s'avérer favorable, il n'en est pas de même sur le plan académique. Il est même possible qu'à certains égards, il soit favorable d'être introverti; le stéréotype de l'étudiant studieux y ressemble passablement (réservé, sobre, discret, solitaire et réfléchi). Cependant, si les Extravertis de notre échantillon manifestent une bonne satisfaction générale, une estime 
d'eux-mêmes supérieure et une intelligence émotionnelle plus élevée, cela n'est pas suffisant pour les faire mieux réussir leurs études en comptabilité ou en marketing.

La satisfaction pour sa vie en général ou pour ses études en particulier ne semblent pas être associées à la Stabilité émotionnelle. Il est possible que le névrosisme entraine l'insatisfaction; nous ne l'avons cependant pas abordé sous cet angle dans la présente enquête.

Un des courants les plus forts en psychologie du travail est celui du travailleur heureux et productif: la satisfaction favoriserait la performance. La cinquième hypothèse que nous avions posée allait dans ce sens. Nous postulions ainsi qu'il existait un lien entre la satisfaction générale et la satisfaction aux études et la performance académique. La deuxième partie

\section{CONCLUSION}

Un étudiant studieux, organisé et méthodique qui envisage la profession de comptable possède selon notre enquête un profil favorable à la réussite académique. Le sentiment d'efficacité (estimation de ses propres capacités à mobiliser sa motivation, les ressources cognitives et les moyens d'action nécessaires pour exercer un contrôle général sur les événements de sa propre vie $^{10}$ ) constitue un facteur de réussite supplémentaire pour cette personne. Plus tard, l'exercice de la profession lui permettra aussi d'utiliser son esprit consciencieux pour atteindre un rendement supérieur au travail : il s'agit donc d'un cheminement cohérent.

En ce qui concerne l'étudiant extraverti, amical, grégaire, ambitieux et loquace, il peut être attiré par le domaine du marketing en pensant pouvoir s'y épanouir. Son trait de personnalité ne le servira possiblement pas pendant ses études. Ce sera le même cas d'ailleurs s'il opte pour une autre orientation: réussir ses études tout en étant sociable et prolixe ne va pas de soi. C'est dommage car si on sélectionne les candidats à des postes en marketing sur la base de leurs résultats académique, ils risquent d'être écarté au profit de candidats plus consciencieux. s'est avérée plutôt juste : les étudiants qui se disaient satisfaits ont montré un niveau de succès académique plus élevé.

Les résultats auxquels nous sommes arrivés recoupent en grande partie ceux de Ones et al. ${ }^{6}$ et de Trapmann et al. ${ }^{17}$. La taille modeste de l'échantillon et l'étroitesse de la mesure de performance académique (note finale plutôt que participation aux discussions en classe, présentations et autres) limite la généralisation possible de nos résultats. Par ailleurs, les items de l'inventaire que nous avons utilisé sont extraits de deux outils différents. Bien que ses qualités métrologiques soient acceptables, il ne s'agit pas d'un instrument de mesure reconnu. Néanmoins, il nous a permis de soulever un petit coin du voile de cette réalité. D'autres études sont nécessaires pour être en mesure de préciser nos résultats et de les généraliser.

\section{BIBLIOGRAPHIE}

${ }^{1}$ Aubin, St. (2008). Prédire la performance au travail par la connaissance de la personnalité. Organisations et territoires, volume 17, numéro 2.

${ }^{2}$ Holland, J. L. (1996). Exploring Careers With a Typology: What We Have Learned and Some New Directions. American psychologist, Vol 51(4), 397-406.

${ }^{3}$ Kuncel, N. R., D.S. Ones et P.R. Sackett (2010). Individual differences as predictors of work, educational, and broad life outcomes. Personality and Individual Differences, 49, 331-336.

${ }^{4}$ Schneider, Benjamin, H. W. Goldstein et D. B. Smith (1995). The ASA framework: an update. Personnel Psychology, vol 48 (4).

${ }^{5}$ Costa, P.T. et R.R. McCrea (1992). Revised NEO Personality Inventory (NEO-P-R) and NEO Fivefactor Inventory (NEO-FFI), Professional Manual. Psychological Assessment Resources: Odessa, Floride.

${ }^{6}$ Ones, D.S., S. Dilchert, C. Viswesvaran et T.A. Judge (2007). In support of Personality Assessment in Organizational Settings. Personnel Psychology, 60, 995-1027.

${ }^{7}$ Barrick, M.R., M.K. Mount et T.A. Judge (2001). Personality and performance at the beginning of the new millenium: What do we know and where do we go next? International journal of Selection and Assessment, 9, 9-30.

${ }^{8}$ Barrick, M.R., M.K. Mount (1991). The Big Five personality dimensions and job performance: A meta- 
analysis. Personnel Psychology, 44, 1-26.

${ }^{9}$ Hough, L.M., D.S. Ones et C. Viswesvaran (1998). Personality correlates of managerial performance constructs. Dans R. Page (éd.). Personality determinants of managerial potential, performance, progression and ascendancy. Actes du Symposium de la $13^{\mathrm{e}}$ conférence annuelle de la Société de Psychologie organisationnelle et Industrielle à Dallas, Texas.

${ }^{10}$ Baudin, N. (2007). Le noyau de l'évaluation de soi : revue de question. Pratiques psychologiques, vol 15 (1), 137-150.

${ }^{11}$ Judge, T.A. et J.E. Bono (2001). Relationship of Core Self-Evaluation Traits -Self-Esteem, Generalized Self-Efficacy, Locus of Control, and Emotional Stability- With Job Satisfaction and Job Performance: A Meta-Analysis. Journal of Applied Psychology, vol 86 (1), 80-92.

${ }^{12}$ Judge, T. A. et A. Erez (2007). Interaction and intersection: The constellation of emotional stability and extraversion in predicting performance. Personnel psychology, vol. 60.

${ }^{13}$ Thoresen, C. J., J. C. Bradley, P. D. Bliese et J. D. Thoresen (2004). The Big Five personality Traits and individual Job performance growth Trajectories in maintenance and transitional Job stages. Journal of Applied Psychology, vol. 89, no 5.

${ }^{14}$ Baumaister, R.F., J.D. Campbell, J.I. Krueger et K.D. Vohs (2003). Does high self-esteem cause better performance, interpersonal success, happiness, or healthier lifestyles? Psychological Science in the Public Interest, vol. 4, no 1.

${ }^{15}$ Tokar, D.M. et J. L. Swanson (1995). Evaluation of the Correspondance between Holland's Vocational Personality Typology and the Five-Factor Model of Personality. Journal of Vocational Behavior, 46, 89-108.

${ }^{16}$ Trapmann, S., B. Hell, J.-O. Hirn et H. Schuler (2007). Merta-Analysis of the Relationship between the Big Five and Academic Success at University. Journal of Psychology, vol 215(2), 132-151.

${ }^{17}$ Chamorro-Premuzic, T. et A. Furhnam (2003). Personality predicts academic performance: Evidence from two longitudinal university samples. Journal of Research in Personality, vol. 37 (4), 319-338

${ }^{18}$ Lounsbury, J.W., E. Sundstrom, J.M. Loveland et L.W. Gibson (2003). Intelligence, «Big Five » poersonality traits, and work drive as predictors of course grade. Personality and Individual Differences, 35, 1231-1239.

${ }^{19}$ Judge, T.A., Erez, A., Bono, J.E., \& Thoresen, C.J. (2003). The Core self-evaluations scale (CSES): development of a measure. Personal Psychology, 56, $303-331$.

${ }^{20}$ Goldberg, L.R., Johnson, J.A., Eber, H.W., Hogan, R., Ashton, M.C., Cloninger, C.R., \& Gough, H.G. (2006). The international personality item pool and the future of public-domain personality measures. Journal of
Research in Personality, 40, $84-96$.

${ }^{21}$ Mount, M.K. et M.R. Barrick (1995). The Big Five personality dimensions: Implications for research and practice in human resources management. Research in Personnel and Human Resources Management, 13, 153-200

${ }^{22}$ El Boukri, Sanaâ (2012). Étude sur les caractéristiques de la personnalité reliées à la performance académique en sciences administratives. Mémoire de maîtrise, inédit, Université du Québec à Chicoutimi.

${ }^{23}$ Judge, T.A., A. Erez, J.E. Bono \& C.J. Thoresen, (2002). Do the traits of self-esteem, neuroticism, locus of control, and generalized self-efficacy-mindicate a common core construct? Journal of Personality and Social Psychology, 83(, 693-710. 


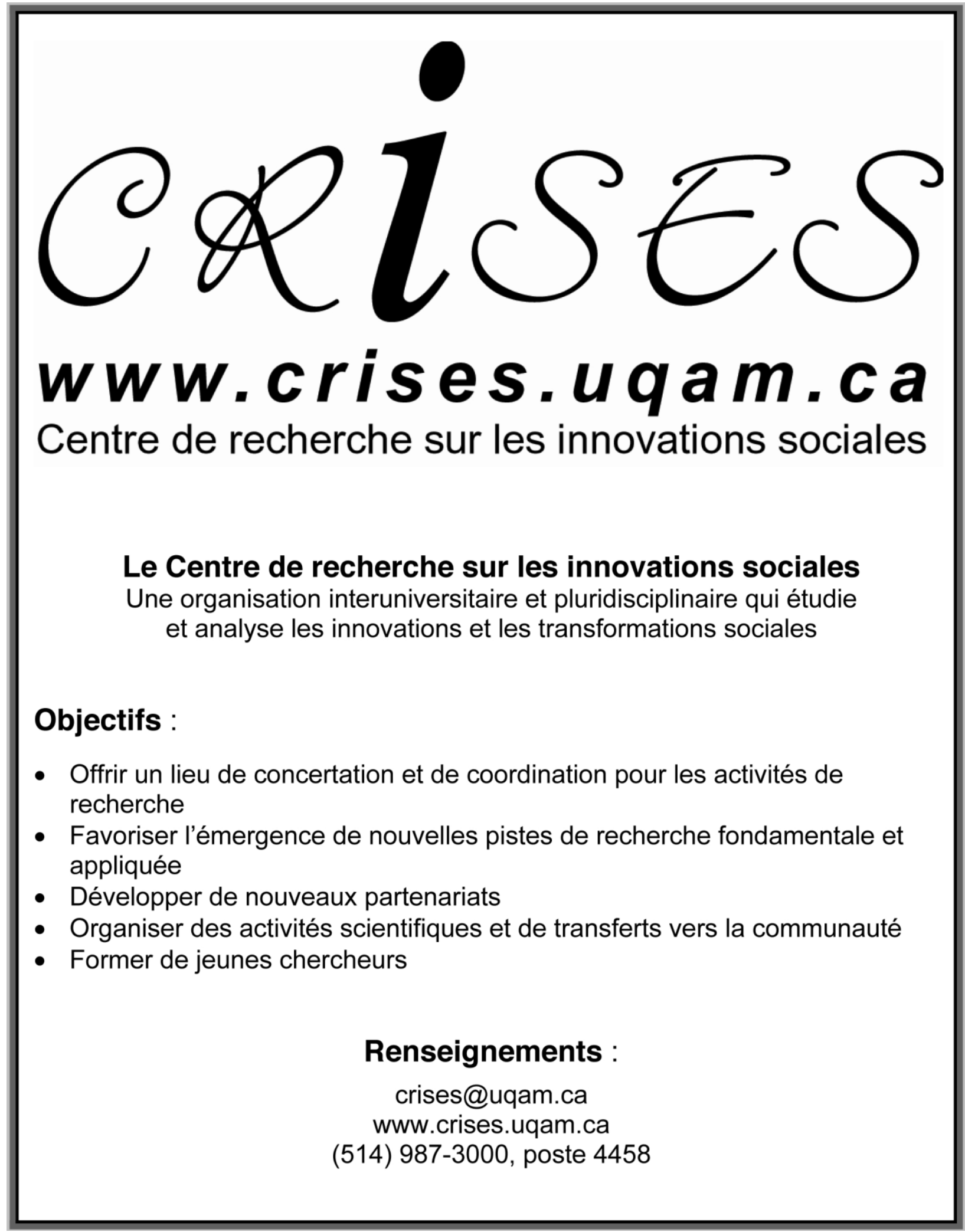

